\title{
Development of a quantitative rapid diagnostic test for multibacillary leprosy using smart phone technology
}

Ludimila Paula Vaz Cardoso ${ }^{1}$, Ronaldo Ferreira Dias ${ }^{2}$, Aline Araújo Freitas ${ }^{1}$, Emerith Mayra Hungria', Regiane Morillas Oliveira', Marco Collovati ${ }^{2}$, Steven G Reed ${ }^{3}$, Malcolm S Duthie ${ }^{3}$ and Mariane Martins Araújo Stefani ${ }^{{ }^{*}}$

\begin{abstract}
Background: Despite efforts to eliminate leprosy as public health problem, delayed diagnosis and disabilities still occur in many countries. Leprosy diagnosis remains based on clinical manifestations and the number of clinicians with expertise in leprosy diagnosis is in decline. We have developed a new immunochromatographic test with the goal of producing a simple and rapid system that can be used, with a minimal amount of training, to provide an objective and consistent diagnosis of multibacillary leprosy.
\end{abstract}

Methods: The test immobilizes two antigens that have been recognized as excellent candidates for serologic diagnosis (the PGL-I mimetic, ND-O, and LID-1), on a nitrocellulose membrane. This allows the detection of specific $\operatorname{lgM}$ and IgG antibodies within 20 minutes of the addition of patient sera. Furthermore, we coupled the NDO-LID ${ }^{\oplus}$ rapid tests with a new cell phone-based test reader platform (Smart Reader ${ }^{\oplus}$ ) to provide objective interpretation that was both quantifiable and consistent.

Results: Direct comparison of serologic responses indicated that the rapid test detected a greater proportion of leprosy patients than a lab-based PGL-I ELISA. While positive responses were detected by PGL-I ELISA in 83.3\% of multibacillary patients and $15.4 \%$ of paucibacillary patients, these numbers were increased to $87 \%$ and $21.2 \%$, respectively, when a combination of the NDO-LID ${ }^{\oplus}$ test and Smart Reader ${ }^{\circledR}$ was used. Among multibacillary leprosy the sensitivity of NDO-LID ${ }^{\circledR}$ test assessed by Smart Reader ${ }^{\circledR}$ was $87 \%(95 \% \mathrm{Cl}, 79.2-92.7 \%)$ and the specificity was 96.1\% (95\% Cl, 91.7- 98.6\%). The positive predictive value and the negative predictive value of NDO-LID ${ }^{\circledR}$ tests were 94\% (95\% Cl, 87.4-97.8\%) and 91.4\% (95\% Cl, 85.9-95.2\%), respectively.

Conclusion: The widespread provision of rapid diagnostic tests to facilitate the diagnosis or prognosis of multibacillary leprosy could impact on leprosy control programs by aiding early detection, directing appropriate treatment and potentially interrupting Mycobacterium leprae transmission.

Keywords: Leprosy, Serologic response, Lateral flow, Brazil

\footnotetext{
*Correspondence: mariane.stefani@pq.cnpq.br

${ }^{1}$ Tropical Pathology and Public Health Institute, Federal University of Goiás, 235th Street, Setor Universitário, 74605-050 Goiânia-Goiás, Brazil

Full list of author information is available at the end of the article
} 


\section{Background}

Leprosy is a dermato-neurological disease that remains an important public health problem in many countries. Patients present with a spectrum of immunological, histological and clinical manifestations that depend on the host immune response to Mycobacterium leprae [1,2]. Clinical manifestations range from the polar lepromatous (LL) forms with high bacterial index (BI), weak $M$. leprae specific cell mediated immunity (CMI) and high antibody titers, to tuberculoid (TT) forms that have low BI, strong CMI and low antibody production. Early diagnosis and treatment are recognized as key elements in the prevention of long-term sequelae associated with leprosy, such as significant impairment of nerve function and deformities, and disabilities are found at greater frequency and severity in patients for whom diagnosis was significantly delayed [3]. In addition, multibacillary (MB) patients with high bacterial burdens are believed to be the primary transmitters of leprosy's etiologic agent, M. leprae, so recognizing and treating infection in these individuals is likely to reduce transmission. Leprosy diagnosis is still, however, based on the recognition of clinical manifestations and reduction in the number of clinicians with expertise in its diagnosis, even in endemic countries, is likely contributing to delayed diagnosis or misdiagnosis $[4,5]$.

There is an expectation that specific and sensitive tests will aid leprosy control programs to further the push toward eliminating this disease. With a goal of developing such tests, antibody responses of leprosy patients to M. leprae proteins and lipids have been extensively investigated, with M. leprae-specific phenolic glycolipid I (PGL-I) antigen representing the most thoroughly investigated to date. Several studies have demonstrated that the anti-PGL-I response is strong among MB patients and reflects the bacillary load, and lateral flow tests have previously been developed to detect the IgM response to PGL-I (ML Flow) and IgM, IgG and IgA antibodies (ML ICA) [6]. Not surprisingly given the limited antibody response of paucibacillary (PB) patients, these tests have limited sensitivity for the detection of $\mathrm{PB}$ patients with low BI $[7,8]$. No objective test for the diagnosis or prognosis of leprosy is, however, currently commercially available.

Our group has previously described novel antigenic proteins that clearly differentiate $\mathrm{MB}$ patients from healthy and M. tuberculosis-infected controls in endemic areas, therefore appearing suitable for the development of serologic tests for leprosy [9-11]. LID-1, a fusion of the ML0405 and ML2331 proteins, has proven to be strongly reactive with $\mathrm{MB}$ patients sera in many geographic locations [10,12-14]. Previous data have suggested that the addition of LID-1 protein to PGL-I could improve sensitivity in a diagnostic test, since some patients lacking antiPGL-I antibodies have antibodies that recognize LID-1, and vice versa [12]. Furthermore, the progression of antibody responses against either PGL-I and LID-1 has been demonstrated to predict the onset of MB leprosy in experimental and clinical surveillance settings [10,14-16].

With a goal of producing a simple and rapid test that can be used with a minimal amount of training to provide an objective and consistent diagnosis of MB leprosy, we developed and evaluated a new rapid test for MB leprosy that incorporates both the LID-1 and PGL-I antigens. These antigens were immobilized on nitrocellulose membranes that permit transfer and detection of specific antibodies in patient's sera. When coupled with a new cell phone-based test reader platform (Smart Reader ${ }^{\circledR}$ application), these tests can provide quantifiable and consistent data to assist in the diagnosis of MB leprosy. The development and widespread provision of rapid tests for $\mathrm{MB}$ leprosy diagnosis, prognosis or classification could have an important impact on leprosy control programs by facilitating/promoting early $\mathrm{MB}$ diagnosis, appropriate treatment and interruption of $M$. leprae transmission.

\section{Methods \\ Study groups}

Between 2006 and 2012, 441 participants were recruited in Goiânia city, Goiás State, Brazil, an endemic area for leprosy, under the approval of the local review board (Comitê de Ética em Pesquisa Humana e Animal/Hospital das Clínicas/Universidade Federal de Goiás) and National Ethics Commission (Comissão Nacional de Ética Pesquisa/ CONEP/Brazil, protocols\#4862/\#12962). All participants (or legal guardians of patients under 18 years) signed an informed consent before blood collection. The test evaluations included five study groups: 1 . Newly diagnosed, untreated MB leprosy patients ( $n=108) ; 2$. Newly diagnosed, untreated PB leprosy patients $(\mathrm{n}=104) ; 3$. Household contacts of $\mathrm{MB}$ and $\mathrm{PB}$ leprosy patients (HHC, $\mathrm{n}=75$ ); 4 . Pulmonary tuberculosis patients with positive bacilloscopy, seronegative for HIV-1/2 and under specific treatment for at least three months (TB, $\mathrm{n}=53) ; 5$. Healthy endemic controls; defined as individuals without previous history of leprosy or TB diagnosis who were not intra-domicilary contacts of leprosy patients (EC, $n=101)$. Participants from both sexes and from all age ranges were included. Leprosy patients were recruited at the main regional public health outpatient clinic (Centro de Referência em Diagnóstico e Terapêutica, Goiânia city, Goiás State) and classified taking into consideration clinical, bacilloscopic and histopathologic data [1]. PB leprosy included TT and borderline-tuberculoid (BT) patients and $\mathrm{MB}$ group included patients in the borderline-borderline (BB), borderline-lepromatous (BL) and LL categories.

The Table 1 describes the main characteristics among the study groups. Among MB leprosy patients 36.1\% 
Table 1 Main characteristics among the study groups recruited in Brazil

\begin{tabular}{|c|c|c|c|c|c|}
\hline & MB & PB & HHC & TB & EC \\
\hline Sex (Female/Male) & $43 / 65$ & $48 / 56$ & $40 / 35$ & $18 / 53$ & $78 / 23$ \\
\hline Age (median range, years) & $48(20-100)$ & $42.5(14-77)$ & $36.5(18-60)$ & $40(17-67)$ & $25(19-66)$ \\
\hline \multicolumn{6}{|l|}{ R \& J classification } \\
\hline LL & $39(36.1 \%)$ & - & - & - & - \\
\hline $\mathrm{BL}$ & 43 (39.8\%) & - & - & - & - \\
\hline BB & $26(24.1 \%)$ & - & - & - & - \\
\hline BT & - & $61(41.4 \%)$ & - & - & - \\
\hline $\mathrm{TT}$ & - & $43(58.7 \%)$ & - & - & - \\
\hline Total/group & 108 & 104 & 75 & 53 & 101 \\
\hline TOTAL $=441$ & & & & & \\
\hline
\end{tabular}

$B B$ borderline-borderline, $B L$ borderline lepromatous, $B T$ borderline- tuberculoid, $E C$ healthy endemic controls, $H H C$ household contacts, $L L$ polar lepromatous, $M B$ multibacillary leprosy, $P B$ paucibacillary leprosy, $R \& J$ Ridley \& Jopling, $T B$ tuberculosis patients, $T T$ polar tuberculoid.

were LL (39/108), 39.8\% were BL (43/108) and 24.1\% were BB (26/108), with a median bacterial index (BI) of 1.0. The PB leprosy group was composed by $41.4 \% \mathrm{TT}$ patients (43/104) and 58.7\% BT patients (61/104). The $\mathrm{HHC}$ group included both contacts of $\mathrm{MB}$ leprosy patients $(80 \% ; 60 / 75)$ and contacts of $\mathrm{PB}$ leprosy patients (20\%; 15/75). The control groups included: TB patients $(\mathrm{n}=53)$ and EC $(\mathrm{n}=101)$.

\section{Enzyme linked immunosorbent assay (ELISA)}

ELISA for the detection of IgM antibodies to PGL-I was performed as previously described $[17,18]$. In brief, the natural trisaccharide-phenyl analog of PGL-I conjugated to bovine serum albumin (NT-P-BSA) was used as antigen and the BSA alone was used as control (Sigma-Aldrich, St. Louis, USA). Serum samples were diluted 1:300 (PBSBSA with $10 \%$ normal goat serum/NGS, Sigma-Aldrich, St. Louis, USA) and tested in duplicate. The reaction was developed by the addition of peroxidase-conjugated anti-human IgM (Cappel/Organon Teknika, Turnhout, Belgium) and 3,3',5,5' -tetramethylbenzidine liquid as substrate system (TMB, Sigma-Aldrich, St. Louis, USA). To control intra- and inter-test variation, a standard reference serum was included in triplicate in each plate. The reaction was quenched by the addition of $2.5 \mathrm{~N} \mathrm{H}_{2} \mathrm{SO}_{4}$ (Sigma-Aldrich, St. Louis, USA) when the optical density (OD) at $450 \mathrm{~nm}$ from the standard reference serum reached a value of 0.7 . The cut-off value for positive results was $\mathrm{OD} \geq 0.250$. Total assay time was 3 hours.

\section{NDO-LID ${ }^{\circledR}$ rapid test}

NDO-LID ${ }^{\bullet}$ rapid test (Orange Life ${ }^{\oplus}$, Rio de Janeiro, Brazil) was developed by impregnating nitrocellulose membranes with ND-O-LID-1, a conjugation of the ND-O (a synthetic mimetic of PGL-I disaccharide) and the LID-1 protein $[10,19,20]$. Panels of well characterized sera from leprosy patients and controls were used to evaluate several prototypes in order to define the final test configuration. The NDO-LID ${ }^{\circ}$ test is provided as a ready-to-use kit and was performed by adding undiluted serum $(10 \mu \mathrm{l})$ and running buffer $(100 \mu \mathrm{l})$ into the sample well, causing the migration of the sample and colloidal gold beads loaded with antiIgG and anti-IgM through the membrane and across a detection window. Interactions with the test and/or control lines are revealed as a red color. Readings were performed after 20 minutes.

Validation of the results required the visualization of a clear control line in the detection window. A positive result was defined by the staining of both the control line and the test line (visual reading scores: $1+/ 1.5+/ 2+$ ); faint or no test line staining was considered as a negative result (Figure 1A and B). Visual readings were performed by three independent readers. In addition, readings were performed using a new Smart Reader ${ }^{\oplus}$ platform (Orange Life $^{\odot}$ ) (Figure 1C) similar to that previously tested with malaria, TB and HIV rapid tests [21,22]. The calculation of Smart Reader ${ }^{\ominus}$ cut-off values was based on the Receiver Operating Curve (ROC), taking into account the visual results of the tests obtained with $\mathrm{MB}$ leprosy patient samples and control samples (TB and EC). Assuming a sensitivity of $87 \%$, as determined by visual readings, the Smart Reader $^{\circledast}$ cut-off was calculated as 9.99 (Figure 2). For data analysis the cut-off for positive results by Smart Reader $^{\oplus}$ was therefore considered as 10.0. Assuming this cut-off, the sensitivity of the test for MB leprosy was $87 \%$ (95\% confidence interval (CI): 79.2 to $92.7 \%)$ and the specificity was $96.1 \%$ (CI 95\%: 91.7 to $98.6 \%$ ), with an area under the curve (AUC) of 0.96 (standard deviation, sd $0.01 ; p<0.0001)$. To validate readings performed 


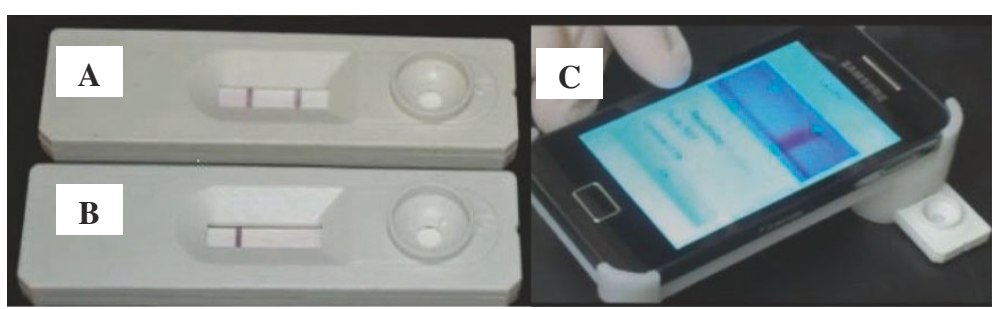

Figure 1 NDO-LID ${ }^{\circledR}$ rapid test. A.: A positive result was defined by the staining of both the control line and the test line (visual reading scores: $1+/ 1.5+/ 2+$; Smart Reader readings $>10$ ). B.: A negative result was defined when a clear control line, but only faint or absent test line, staining was observed. C.: Readings and objective scoring were achieved using a new Smart Reader ${ }^{\circledast}$ platform.

by Smart Reader ${ }^{\circledR}$ for NDO-LID ${ }^{\bullet}$ test, the positive predictive value (PPV) and the negative predictive value (NPV) were calculated as $94 \%$ and $91.4 \%$, respectively.

\section{Statistical analysis}

Frequencies, median, mean of OD values and graphics were obtained (GraphPad Prism version 5). Statistical significance was assessed using Kruskal-Wallis one-way analysis of variance for comparison of multiple groups and by Mann-Whitney $U$ test for comparison between two groups. Results were considered statistically significant when $p$ values $<0.05$ were obtained.

The concordance between results of the ELISA and NDO-LID ${ }^{\circ}$ tests was determined by agreement and kappa values (K) with 95\% CI and sd value were calculated (Statistical Package for the Social Sciences, SPSS version 13.0). The following interpretation of agreement was used for the kappa values: low (0-0.5), moderate (0.51-0.75) and excellent (0.76-1.0) following established guidelines [23]

The accuracy of the NDO-LID ${ }^{\odot}$ tests was evaluated by ROC (GraphPad Prism version 5) considering the AUC,

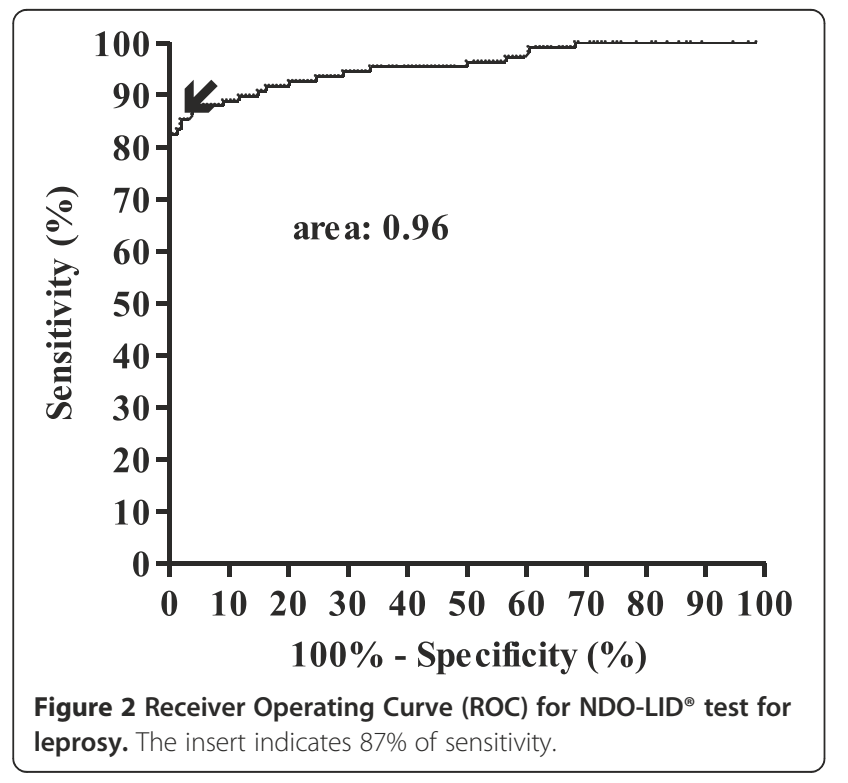

in which a test that does not discriminate healthy individuals from patients, gives a value of AUC of 0.5 (null hypothesis). In our case the test discriminates MB leprosy from the other groups. A value above 0.7 is considered a satisfactory performance [24]. Moreover, the sensitivity, specificity and the PPV and NPV values were determined with 95\% CI (GraphPad Prism version 5).

\section{Results}

Detection of MB patients by serologic tests

The anti-PGL-I ELISA positive rate among the MB leprosy patients tested was determined to be $83.3 \%$ (90/108; median $\mathrm{OD}=0.766$, range 0.285 to 2.755 ). As expected, seropositivity varied across the different $\mathrm{MB}$ leprosy categories: $92.3 \%$ for LL patients $(36 / 39$; median OD = 0.756 , range 0.333 to 2.688$), 69.8 \%$ for $\mathrm{BL}$ patients (30/43; median $\mathrm{OD}=0.754$, range 0.313 to 2.566 ) and $92.3 \%$ for $\mathrm{BB}$ patients $(24 / 26$; median $\mathrm{OD}=0.772$, range 0.285 to 2.755) (Figure 3A).

Among these same MB leprosy patients, the seropositive rate in NDO-LID ${ }^{\circ}$ test was found to be slightly higher at $87 \%$ (94/108), with similar results obtained by both visual reading and the Smart Reader $^{\odot}$ platform (Figures 3B and $\mathrm{C}$ ). These results demonstrate concordance at both performance and signal intensity of laboratory-based assays with the rapid test/Smart Reader ${ }^{\circledR}$ application. A decrease in the score of visual reading was observed across the spectrum of the disease from the MB (LL, BL and $\mathrm{BB}$ ) to $\mathrm{PB}$ (TT, BT) pole when using the NDO-LID ${ }^{\circ}$ tests (Figure $3 \mathrm{~B}$ ). Positive rates ranged from $97.4 \%$ for $\mathrm{LL}$ $(38 / 39$; median $=58$, range 11 to 92.5$), 83.7 \%$ among $\mathrm{BL}$ $(36 / 43$; median $=21.9$, range 15.1 to 90.1$)$ and $76.9 \%$ among BB leprosy (20/26; median $=44.9$, range 10 to 44.9). Thus, similar declines in signal intensity across the clinical spectrum were measured by the Smart Reader $^{\oplus}$ application, although a far greater range of signal intensity was captured by the electronic reader (Figure 3C). Although not statistically significant, the NDO-LID ${ }^{\circ}$ rapid test actually slightly outperformed laboratory-based anti-PGL-I ELISA, suggesting an added benefit by including the LID-1 protein and indicating utility for the clear and simple detection of MB leprosy patients. 


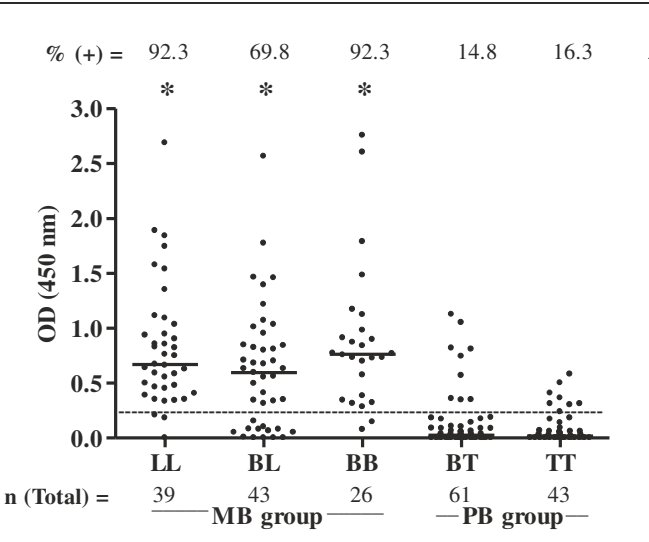

\section{A}
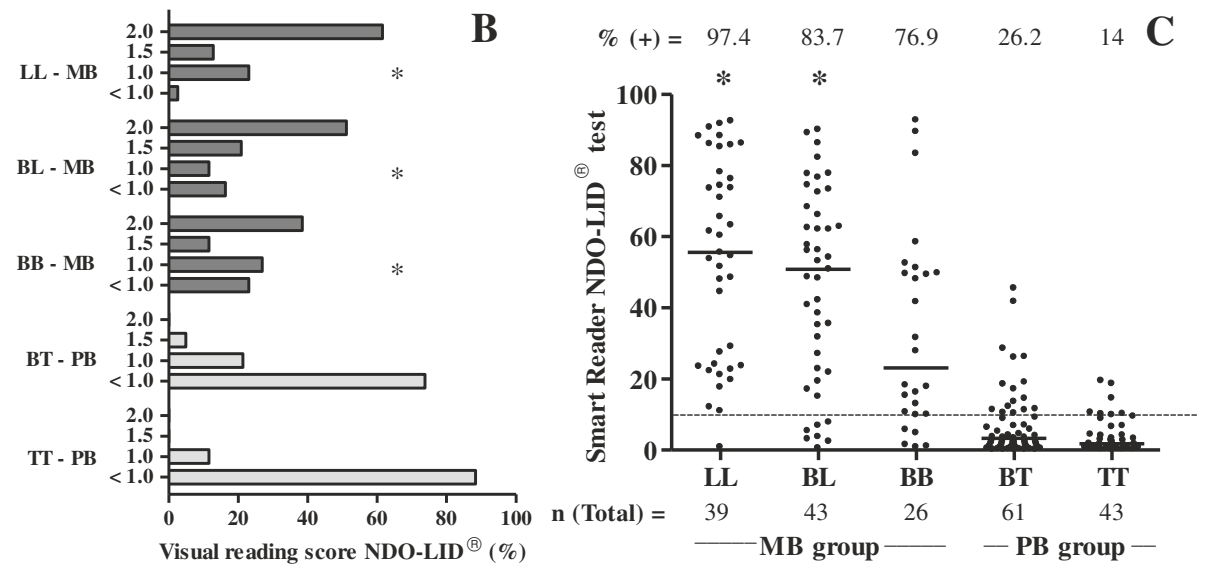

Figure 3 PGL-I ELISA and NDO-LID ${ }^{\circledR}$ tests assessed among multibacillary (MB) and paucibacillary (PB) leprosy groups. The number above each data set is the percentage of positive results and the number below each data set represents the total number of participants of each group. A.: IgM PGL-I ELISA test: each point represents the mean value of optical density (OD) of individual serum samples. The median OD value of each group is represented by the horizontal line. The traced horizontal line is the cut-off $(O D \geq 0.250) .^{*}=p<0.05$ ( $L L$ versus BT and TT; BL versus BT and TT; $\mathrm{BB}$ versus $\mathrm{BT}$ and TT). B.: Visual reading of NDO-LID ${ }^{\oplus}$ test scored ${ }^{*}=p<0.05$. C.: NDO-LID ${ }^{\oplus}$ test assessed by Smart Reader ${ }^{\oplus}$ platform: each point represents the Smart Reader ${ }^{\oplus}$ result of an individual serum sample. The horizontal line represents the median of the Smart Reader ${ }^{\otimes}$ reading for each group and the traced horizontal line is the cut-off ( $\geq 10) .{ }^{*}=p<0.05$ ( $L L$ versus BT and TT; BL versus BT and TT; BB versus BT and TT).

\section{Enhanced detection of PB leprosy by NDO-LID ${ }^{\circledR}$ test} As expected, when samples from PB leprosy patients were evaluated, the anti-PGL-I ELISA was found to be positive in only a minor subset, and with a reduced signal intensity compared to $\mathrm{MB}$ patients. An overall rate of $15.4 \%$ positive responders were found for $\mathrm{PB}$ patients $(16 / 104$; median $\mathrm{OD}=0.454$, range 0.300 to 1.126$)$, ranging from $14.8 \%$ among $\mathrm{BT}$ patients (9/61; median $\mathrm{OD}=0.359$, range 0.346 to 1.126 ) to $16.3 \%$ for TT patients $(7 / 43$; median $\mathrm{OD}=0.408$, range 0.300 to 0.581$)$ (Figure 3A).

As with the evaluations in $\mathrm{MB}$ patients, an increased number of $\mathrm{PB}$ patients was detected by the NDO-LID ${ }^{\circ}$ test. The NDO-LID ${ }^{\curvearrowright}$ rapid test was visually positive for $20.2 \%$ of PB leprosy patients (21/104): $26.2 \%$ for BT patients $(16 / 61)$ and $11.6 \%$ for TT patients $(5 / 43)$ (Figure 3B). NDO-LID ${ }^{\bullet}$ results assessed by Smart Reader ${ }^{\circledR}$ platform demonstrated a $21.2 \%$ positivity rate for $\mathrm{PB}$ leprosy patients (22/104): 26.2\% among BT patients $(16 / 61$; median $=15.85$, range 10.4 to 45.5$)$ and $14 \%$ among TT patients $(6 / 43$; median $=12.6$, range 10.2 to 19.5) (Figure 3C). Although these data indicate limited utility of the serological assays for detection of $\mathrm{PB}$ cases, they do indicate that some $\mathrm{PB}$ patients can actually be detected by this simple rapid test. This was particularly true for BT patients, where the rapid test almost doubled the detection rate over PGL-I ELISA.

\section{Seroreactivity among $\mathrm{HHC}$ and control groups}

$\mathrm{HHC}$ of untreated MB patients represent an important study group as they are believed to be exposed to $M$. leprae at a higher frequency than the general population and seropositive contacts are considered to be at higher risk of progressing towards $\mathrm{MB}$ disease. In this study we tested $\mathrm{HHC}$ of both $\mathrm{PB}$ and $\mathrm{MB}$ patients. Overall, anti-PGL-I ELISA positivity among HHC was 
low $(2.7 \%, 2 / 75)$ : one contact from $\mathrm{MB}$ and another contact from $\mathrm{PB}$ leprosy patient $(\mathrm{OD}=0.308$ and 0.289 , respectively) (Figure 4A).

For the NDO-LID ${ }^{\circ}$ test an identical rate of seropositivity was detected in $\mathrm{HHC}$ by visual reading and by Smart Reader $(5.3 \%$; 4/75); detecting 2 additional individuals along with both that were anti-PGL-I positive by ELISA (one of them $\mathrm{HHC}$ of MB patient and the other $\mathrm{HHC}$ of $\mathrm{PB}$ patient) (Figures $4 \mathrm{~B}$ and $\mathrm{C}$ ). For the two $\mathrm{MB}$ HHC seropositive, the Smart Reader readings were 16.8 and 21.8 and for the two PB HHC seropositive, the Smart Reader ${ }^{\circ}$ readings were 11.4 and 14.4, respectively.

Among TB patients the overall anti-PGL-I ELISA positivity was $3.8 \%(2 / 53$; OD $=0.301$ and 0.302$)$ and no responses were observed among EC (Figure 4A). For TB patients tested by NDO-LID ${ }^{\circ}$ the same positive rate was obtained by visual reading and by Smart Reader ${ }^{\circ}$ readings $(5.7 \%, 3 / 53)$. For EC $4 \%(4 / 101)$ were positive by visual reading, while $3 \%$ were positive using the Smart Reader $^{\circ}(3 / 101, p>0.05)$ (Figures $4 \mathrm{~B}$ and $\mathrm{C}$ ). Together, these data indicate that, despite possible cross-reactivity with other mycobacteria circulating in the test populations, the rapid test is highly specific for leprosy.

\section{NDO-LID ${ }^{\circledR}$ rapid test enhances patient detection over anti-PGL-I ELISA}

For the NDO-LID ${ }^{\circ}$ test adopting the Smart Reader ${ }^{\circ}$ cut-off $\geq 10$, a high degree of agreement (99.1\%; 437/441) was observed between the visual reading and the Smart Reader $^{\circledast}$ (kappa value $\left.=0.98 ; \mathrm{sd}=0.011\right)$ (Table 2). The four discordant cases were a single TT patient (negative by visual reading and Smart Reader ${ }^{\oplus}$ positive $=10.2$ ), a single EC (positive by visual reading and Smart Reader ${ }^{\circ}$ negative) and two TB patients (one positive by visual reading, negative in Smart Reader ${ }^{\circ}$ and one negative by visual reading and Smart Reader ${ }^{\bullet}$ positive $=10.8$ ).

In $90.9 \%(401 / 441)$ of the samples anti-PGL-I ELISA and NDO-LID ${ }^{\circ}$ results were concordant (either positive or negative; kappa value $=0.8$; sd $=0.034$ ) (Table 3 ). Among these, 98 were positive in both tests (86 MB leprosy; $10 \mathrm{~PB}$;
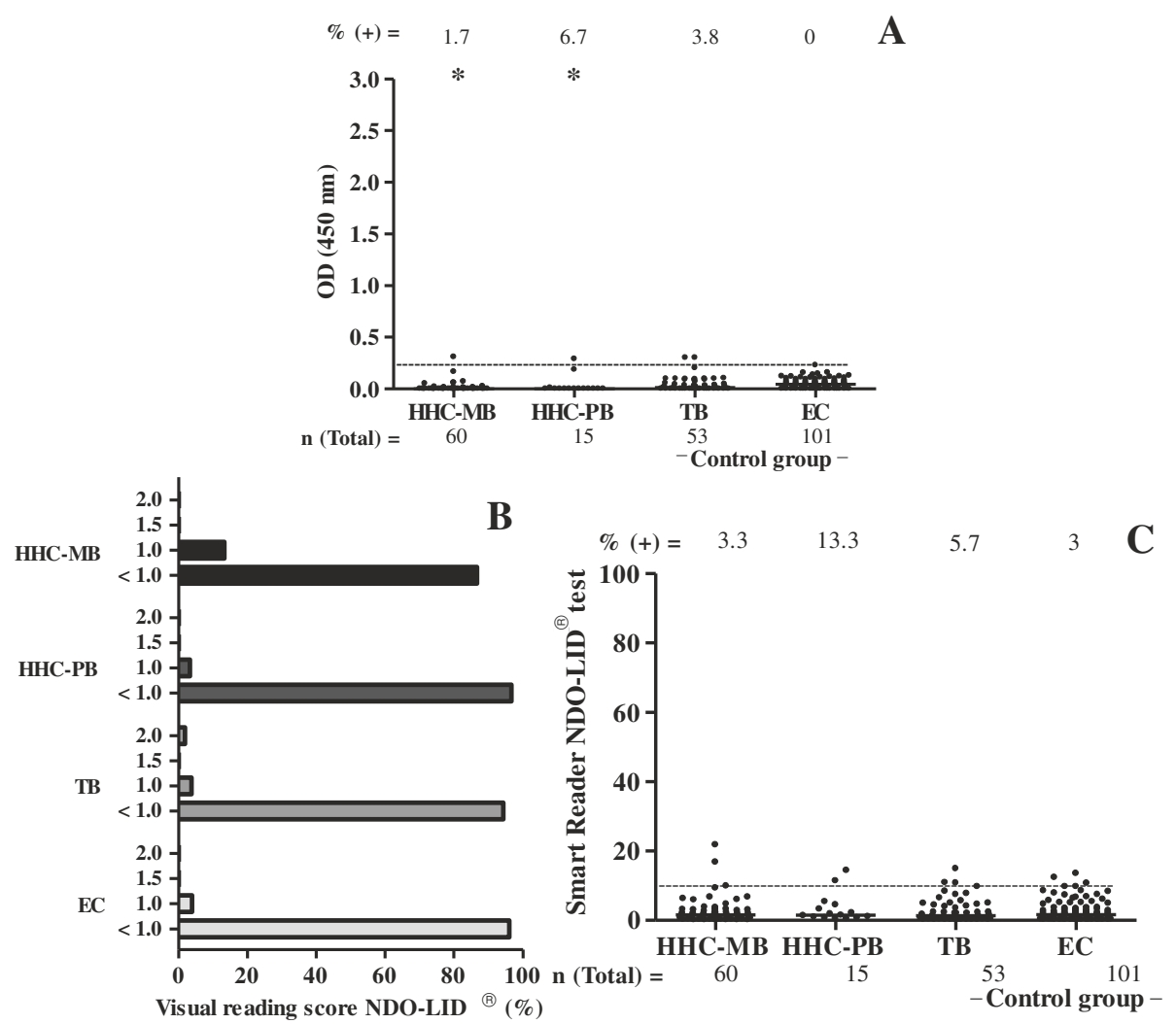

Figure 4 PGL-I ELISA and NDO-LID ${ }^{\otimes}$ tests assessed among household contacts (HHC), tuberculosis (TB) patients and healthy endemic control (EC) groups. The number above each data set is the percentage of positive results and the number below each data set represents the total number of participants of each group. A.: IgM PGL-I ELISA test: each point represents the mean value of optical density (OD) of individual serum samples. The median OD value of each group is represented by the horizontal line. The traced horizontal line is the Cut-off (OD $\geq 0.250)$. ${ }^{*}=p<0.05$ (HHC-MB versus TB and EC; HHC-PB versus EC). B.: Visual reading of NDO-LID ${ }^{\oplus}$ test scored. C.: NDO-LID ${ }^{\oplus}$ test assessed by Smart Reader ${ }^{\oplus}$ platform: each point represents the Smart Reader ${ }^{\circledR}$ result of an individual serum sample. The horizontal line represents the median of the Smart Reader ${ }^{\oplus}$ reading for each group and the traced horizontal line is the cut-off ( $\left.\geq 10\right)$. 
Table 2 NDO-LID ${ }^{\circledR}$ test results: comparison between visual reading and Smart Reader ${ }^{\circledR}$

\begin{tabular}{|c|c|c|c|c|}
\hline \multicolumn{2}{|l|}{ NDO-LID ${ }^{\circledast}$ test } & \multicolumn{3}{|c|}{ Visual reading } \\
\hline & & Positive & Negative & Total \\
\hline \multirow[t]{3}{*}{ Smart Reader ${ }^{\circledast}$ platform } & Positive & 124 & 3 & 127 \\
\hline & Negative & 1 & 313 & 314 \\
\hline & Total & 125 & 316 & 441 \\
\hline
\end{tabular}

$2 \mathrm{HHC}$ ) and 303 samples were negative in both tests. The discordant rate observed between the NDO-LID ${ }^{\circ}$ and antiPGL-I ELISA test was 9.1\% (40/441). A total of 28 NDOLID $^{\circ}$ positive results were revealed among 331 anti-PGL-I ELISA negative samples: 8 from MB leprosy patients, 11 from PB leprosy, 2 from HHC, 3 from TB patients, 4 from EC. Conversely, only 12 negative NDO-LID ${ }^{\circ}$ tests were found among anti-PGL-I positive samples: 4 from $\mathrm{MB}$ leprosy (median anti-PGL-I OD $=0.539$, range 0.315 0.757), 6 from $\mathrm{PB}$ leprosy patients (median $\mathrm{OD}=0.575$, range $0.300-0.409)$ and 2 from $\mathrm{TB}$ patients $(\mathrm{OD}=0.301$ and 0.302).

\section{Discussion}

Although leprosy remains an important public health problem in many countries, including Brazil, diagnosis is still based on the recognition of clinical manifestations by highly skilled clinicians [25]. A major concern in the widespread control efforts for leprosy is, however, that even in endemic regions the number of such clinicians that can actually recognize early signs or provide a confident differential diagnosis from other skin diseases is limited and in decline $[4,26]$. Tests that could be conducted on individuals with even a minimal suspicion of leprosy by general health workers, in conjunction with clinical exam by physicians, could facilitate referrals or initiate increased levels of monitoring.

The application of a rapid test for leprosy needs to take into account both the spectral nature of the disease and the immunological dichotomy in which at one pole PB patients develop weak (or absent) antibody responses while at the opposite pole MB patients develop a robust humoral immune response. The PGL-I and LID-1 antigens have been among the most reactive antigens tested in different populations, including multiple sites in Brazil, the Philippines, Japan, Nepal, Venezuela, Bangladesh, and India, suggesting utility in a global context $[10,12,13]$. As

Table 3 NDO-LID ${ }^{\oplus}$ and IgM anti-PGL-I ELISA results

\begin{tabular}{lcccc}
\hline & & \multicolumn{3}{c}{ NDO-LID $^{\circledR}$ test } \\
\cline { 3 - 5 } & & Positive & Negative & Total \\
\hline PGL-I ELISA & Positive & 98 & 12 & 110 \\
& Negative & 28 & 303 & 331 \\
& Total & 128 & 313 & 441 \\
\hline
\end{tabular}

expected, the majority of MB leprosy patients were identified by serological assays, yielding higher responses than PB patients. Some studies have reported a moderate frequency of anti-PGL-I antibodies and anti-IgG antibodies to recombinant $M$. leprae proteins in PB leprosy patients (range 15 to $40 \%$ ) $[8,10,11]$. An important limitation that must therefore be recognized for any serological test for leprosy is that their ability to detect PB patients may be limited. Given the results of anti-PGL-I ELISA, the seropositivity among $\mathrm{PB}$ leprosy patients by NDO-LID ${ }^{\circ}$ rapid test in this study can be considered higher than expected (20.2\%). The intra-domiciliary contacts of leprosy patients, especially MB patients, likely have a markedly increased rate of infection and absolutely have an increased risk of developing MB leprosy compared to the general population; among anti-PGL-I positive contacts this elevated risk is even higher $[27,28]$. We observed low response rates among contacts from both $\mathrm{MB}$ and $\mathrm{PB}$ leprosy patient groups, suggesting that positive tests may be indicative of symptomatic leprosy or asymptomatic $M$. leprae infection and not simply exposure. It should be noted that, even in a groups of 75 contacts and over 100 healthy controls as tested here, only few actual cases are predicted to emerge. Laboratory-based studies have already been used to alert surveillance teams to individuals that have developed clinical symptoms subsequent to strong results in LID-1 ELISA, at levels that are readily detected in the rapid test $[14,16]$. Expanded evaluations, either through bridging studies of archived sera or preferably through 'live' testing as a component of sustained monitoring campaigns, are needed to better indicate the potential benefits of incorporating such a test by leprosy control programs in endemic countries. The rapidity and ease-of-use of the test also suggests that expanded screening within patient populations and their contacts including individuals younger than 15 years could be readily achievable and would provide important information for leprosy control programs.

For operational purposes a simplified leprosy classification system based on the number of skin lesions defines that PB leprosy presents with up to five lesions and usually include TT and BT forms whereas MB leprosy presents with more than five skin lesions and usually comprise LL, BL and BB forms [29]. However, the accuracy of this classification criterion is limited and may lead to misclassification and inadequate treatment [30,31]. Differentiation to the general classifications orients specific multidrug therapy (MDT) which consists of either six doses of rifampim and dapsone for PB leprosy or twelve doses of rifampim, dapsone and clofazimine for MB patients. Several studies have identified that antiPGL-I responses and the ML Flow rapid test can help in the classification of $\mathrm{MB}$ and $\mathrm{PB}$ leprosy patients to orient the choice of treatment $[6,8,32,33]$. The rapid test 
that we have developed retains the ability to aid MB and PB leprosy classification, although the ability to 'diagnose' PB leprosy remains limited. This is not surprising given the generally low anti- $M$. leprae antibody responses of PB patients. It has been previously reported that serum antibody responses to PGL-I and to LID-1 antigens decline upon MDT [15,34]. Importantly, given the enhanced fidelity of rapid test interpretation when incorporated with the Smart Reader ${ }^{\circ}$, monitoring of patients on treatment could potentially reveal complications such as relapses (or reinfection), providing improved patient care and management.

Typically, lateral flow-based rapid test have been scored subjectively on a grading system after visual inspection. Even with comparative examples, it is difficult to achieve consistency in these readings unless the reader(s) has garnered a large amount of experience. Additionaly, results from any diagnostic test would ideally be blinded from the clinical evaluation but in most settings, such as rural settings with limited resources and personnel or busy urban clinics, this is difficult to achieve. Considering the possibility of incorrect or bias in human reading of rapid tests in field conditions, an important innovation presented in this study is the use of a digital and automated rapid test Smart Reader application. At the sole additional expense of a standard cell phone, this provides a means to generate controlled and consistent results across diverse endemic settings. As an extra layer of quality control and patient care, this compact pocket-size digital reader permits the transmission of digital data and other related information (e.g., demographic data) to a cloud, allowing the information to be downloaded for record keeping and/or be transferred to off-site experts for a rapid second opinion. This new technological strategy also allows real time spatio-temporal analysis of the disease prevalence and incidence in the region under evaluation.

For any serological test, both sensitivity and specificity represent the main parameters which were evaluated in this study by the inclusion of well characterized newly diagnosed, untreated leprosy patients (clinical, histopathological and microbiological features) and controls from an endemic area (EC and patients with TB). Among MB leprosy the sensitivity of NDO-LID ${ }^{\circ}$ test assessed by Smart Reader ${ }^{\circ}$ was $87 \%$ (95\% CI, 79.2-92.7\%) and the specificity was $96.1 \%$ (95\% CI, 91.7- 98.6\%). The PPV and the NPV of NDO-LID ${ }^{\circ}$ tests were $94 \%$ (95\% CI, 87.4-97.8\%) and $91.4 \%$ (95\% CI, 85.9-95.2\%), respectively. Potential cross-reactivity is an important issue to be considered in the development of a new serologic test, particularly for countries with a high incidence of TB, high BCG vaccination coverage and high levels of exposure to environmental mycobacteria $[35,36]$. Using NDO-LID ${ }^{\circ}$ test low positivity among healthy EC was observed. This is an important data since for most individuals from an endemic country, such as Brazil, there is a high chance of exposure to $M$. leprae throughout life especially in the highly endemic Northeast, North and Central Western regions, the latter representing the area where participants were recruited. Moreover the geographical region where participants were recruited is characterized by a very high BCG vaccination coverage (close to $100 \%$ ). The low NDO-LID ${ }^{\circ}$ positivity among $\mathrm{TB}$ and EC indicates that previous BCG vaccination, at least within the age range of participants is not associated with cross reactivity in the new rapid test [6,37]. The low seropositivity among TB patients observed with NDO-LID ${ }^{\circ}$ test indicates that cross-reaction between $M$. tuberculosis antigens is not a significant concern. For the 3 (out of 53) TB patients with positive NDO-LID ${ }^{\circ}$ test, subclinical infection or exposure to $M$. leprae cannot not be excluded. In general, the low positivity among control groups further supports the use of NDO-LID ${ }^{\circ}$ test in an endemic country as Brazil.

\section{Conclusions}

The new NDO-LID ${ }^{\circ}$ rapid test presented in this study represents an important development in leprosy control, capable of providing a practical means to assist in the rapid, consistent and quantitative detection of MB leprosy. Considering the need for a rapid test in leprosy endemic countries, broader validation studies are being developed to evaluate performance under field conditions, including the direct use of finger prick blood samples to demonstrate its utility as a true point of care test. The NDO-LID ${ }^{\circ}$ rapid test could ultimately aid leprosy control programs by allowing greater numbers of individuals to be simply tested at a greater frequency, and could be instrumental in the identification and prompt treatment of MB patients. Together, such applications of the test could help reach the WHO recommendation of early diagnosis to interrupt the transmission of $M$. leprae and further reduce leprosy case numbers.

\section{Abbreviations}

AUC: Area under the curve; BI: Bacterial index; BB: Borderline-borderline; BL: Borderline-lepromatous; BT: Borderline-tuberculoid; BSA: Bovine serum albumin; CMI: Cell mediated immunity; Cl: Confidence interval; ELISA: Enzyme linked immunosorbent assay; EC: Healthy endemic controls; HHC: Household contacts; K: Kappa values; LL: Lepromatous; M. leprae: Mycobacterium leprae;

M. tuberculosis: Mycobacterium tuberculosis; MB: Multibacillary; MDT: Multidrug therapy; NPV: Negative predictive value; NGS: Normal goat serum; OD: Optical density; PB: Paucibacillary; PGL-I: Phenolic glycolipid I; PPV: Positive predictive value; ROC: Receiver operating curve; sd: Standard deviation; SPSS: Statistical package for the social sciences; ND-O: Synthetic mimetic of PGL-I disaccharide; TT: Tuberculoid; TB: Tuberculosis; TMB: 3,3',5,5'-tetramethylbenzidine.

\section{Competing interests}

The authors declare that they have no competing interests. Marco Collovati is the owner of Orange Life ${ }^{\circledR}$ (Rio de Janeiro/Brazil), the company producing and marketing the NDO-LID ${ }^{\oplus}$ rapid test and Ronaldo Ferreira Dias, Orange Life $^{\oplus}$ employee. 


\section{Authors' contributions}

LPVC participated in the study designs, carried out the immunoassays, performed the statistical analysis and drafted the manuscript. RFD participated in the study design and carried out the immunoassays. AAF, EMH and RMO participated in the field work and carried out the immunoassays. MC participated in the study design. SGR participated in the study design and reviewed the manuscript. MSD conceived, participated in the study design and contributed to write the manuscript. MMAS conceived, designed the study, coordinated the field work, analyzed the data and contributed to write the manuscript. All authors read and approved the final manuscript.

\section{Acknowledgments}

This study was supported by American Leprosy Missions, Renaissance Health Service Corporation and PRONEX/FAPEG/CNPq-07/2009. Dr. Mariane M. A. Stefani is a recipient of a fellowship from CNPq (grant \# 304869/2008-2) and Dr. Ludimila Cardoso was supported by a fellowship from CAPES (grant\#02479/09-5). We thank Dr. Fujiwara (Institute for Natural Science, Nara University, Nara, Japan) for kindly providing the NTP-BSA antigen.

\section{Author details}

${ }^{1}$ Tropical Pathology and Public Health Institute, Federal University of Goiás, 235th Street, Setor Universitário, 74605-050 Goiânia-Goiás, Brazil. ²Orange Life, Rio de Janeiro, Brazil. ${ }^{3}$ Infectious Disease Research Institute, Seattle, USA.

\section{Received: 28 May 2013 Accepted: 17 October 2013}

Published: 23 October 2013

\section{References}

1. Ridley DS, Jopling WH: Classification of leprosy according to immunity. A five-group system. Int J Lepr Other Mycobact Dis 1966, 34:255-273.

2. Modlin RL: The innate immune response in leprosy. Curr Opin Immunol 2010, 22(1):48-54.

3. Scollard DM: The biology of nerve injury in leprosy. Lepr Rev 2008, 79(3):242-253.

4. Siddiqui MR, Velidi NR, Pati S, Rath N, Kanungo AK, Bhanjadeo AK, Rao BB, Ojha BM, Krishna Moorthy K, Soutar D, Porter JD, Ranganadha Rao PV: Integration of leprosy elimination into primary health care in orissa, India. PLoS One 2009, 18(12):e8351.

5. Correa RDA, Aquino DM, Caldas AJ, Amaral DK, França FS, Mesquita ER: Epidemiological, clinical, and operational aspects of leprosy patients assisted at a referral service in the state of Maranhão, Brazil. Rev Soc Bras Med Trop 2012, 45(1):89-94.

6. Stefani MM, Grassi AB, Sampaio LH, Sousa AL, Costa MB, Scheelbeek $P$, Neupane KD, Hagge DA, Macdonald M, Cho SN, Oskam L, Bührer-Sékula S: Comparison of two rapid tests for anti-phenolic glycolipid-I serology in Brazil and Nepal. Mem Inst Oswaldo Cruz 2012, 107(Suppl):124-131.

7. Klatser PR, Cho SN, Brennan PJ: The contribution of serological tests to leprosy control. Int J Lepr Other Mycobact Dis 1996, 64(Suppl):S63-S67.

8. Bührer-Sékula S, Smits HL, Gussenhoven GC, van Leeuwen J, Amador S, Fujiwara T, Klatser PR, Oskam L: Simple and fast lateral flow test for classification of leprosy patients and identification of contacts with high risk of developing leprosy. J Clin Microbiol 2003, 41(5):1991-1995.

9. Reece ST, Ireton G, Mohamath R, Guderian J, Goto W, Gelber R, Groathouse N, Spencer J, Brennan P, Reed SG: ML0405 and ML2331 are antigens of Mycobacterium leprae with potential for diagnosis of leprosy. Clin Vaccine Immunol 2006, 13(3):333-340.

10. Duthie MS, Goto W, Ireton GC, Reece ST, Cardoso LP, Martelli CM, Stefani MM Nakatani M, de Jesus RC, Netto EM, Balagon MV, Tan E, Gelber RH, Maeda Y, Makino M, Hoft D, Reed SG: Use of protein antigens for early serological diagnosis of leprosy. Clin Vaccine Immunol 2007, 14:1400-1408.

11. Sampaio LH, Stefani MM, Oliveira RM, Sousa AL, Ireton GC, Reed SG, Duthie MS: Immunologically reactive $M$. leprae antigens with relevance to diagnosis and vaccine development. BMC Infect Dis 2011, 11:26-37.

12. Hungria EM, Oliveira RM, Souza AL, Costa MB, Souza VN, Silva EA, Moreno FR, Nogueira ME, Costa MR, Silva SM, Bührer-Sékula S, Reed SG, Duthie MS, Stefani MM: Seroreactivity to new Mycobacterium leprae protein antigens in different leprosy-endemic regions in Brazil. Mem Inst Oswaldo Cruz 2012, 107(Suppl):S104-S111.

13. Rada E, Duthie MS, Reed SG, Aranzazu N, Convit J: Serologic follow-up of lgG responses against recombinant mycobacterial proteins ML0405,
ML2331 and LID-1 in a leprosy hyperendemic area in Venezuela. Mem Inst Oswaldo Cruz 2012, 107(Suppl):90-94.

14. Qiong-Hua P, Zhong-Yi Z, Jun Y, Yan W, Lian-Chao Y, Huan-Ying L, Reed SG, Duthie MS: Early Revelation of Leprosy in China by Sequential Antibody Analyses with LID-1 and PGL-I. J Trop Med 2013, 2013:352689.

15. Duthie MS, Truman RW, Goto W, O'Donnell J, Hay MN, Spencer JS, Carter D, Reed SG: Insight toward early diagnosis of leprosy through analysis of the developing antibody responses of Mycobacterium leprae-infected armadillos. Clin Vaccine Immunol 2011, 18(2):254-259.

16. Spencer JS, Duthie MS, Geluk A, Balagon MF, Kim HJ, Wheat WH, Chatterjee D, Jackson M, Li W, Kurihara JN, Maghanoy A, Mallari I, Saunderson P, Brennan PJ, Dockrell HM: Identification of serological biomarkers of infection, disease progression and treatment efficacy for leprosy. Mem Inst Oswaldo Cruz 2012, 107(Suppl):79-89.

17. Brett SJ, Payne SN, Gigg J, Burgess P, Gigg R: Use of synthetic glycoconjugates containing the Mycobacterium leprae specific and immunodominant epitope of phenolic glycolipid I in the serology of leprosy. Clin Exp Immunol 1986, 64(3):476-483.

18. Bührer-Sékula S, Sarno EN, Oskam L, Koop S, Wichers I, Nery JA, Vieira LM, de Matos HJ, Faber WR, Klatser PR: Use of ML dipstick as a tool to classify leprosy patients. Int J Lepr Other Mycobact Dis 2000, 68:456-463.

19. Cho SN, Fujiwara T, Hunter SW, Rea TH, Gelber RH, Brennan PJ: Use of an artificial antigen containing the 3,6-di-O-methyl-beta-D-glucopyranosyl epitope for the serodiagnosis of leprosy. J Infect Dis 1984, 150(3):311-322.

20. Fujiwara T, Hunter SW, Cho SN, Aspinall GO, Brennan PJ: Chemical synthesis and serology of disaccharides and trisaccharides of phenolic glycolipid antigens from the leprosy bacillus and preparation of a disaccharide protein conjugate for serodiagnosis of leprosy. Infect Immun 1984, 43(1):245-252.

21. Lee DS, Jeon BG, Ihm C, Park JK, Jung MY: A simple and smart telemedicine device for developing regions: a pocket-sized colorimetric reader. Lab Chip 2011, 7:120-126.

22. Mudanyali O, Dimitrov S, Sikora U, Padmanabhan S, Navruz I, Ozcan A: Integrated rapid-diagnostic-test reader platform on a cellphone. Lab Chip 2012, 7(15):2678-2686.

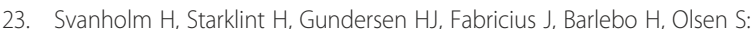
Reproducibility of histomorphologic diagnoses with special reference to the Kappa statistic. APMIS 1989, 97:689-698.

24. Beck JR, Shultz EK: The use of relative operating characteristic (ROC) curves in test performance evaluation. Arch Pathol Lab Med 1986, 110(1):13-20. Erratum in: Arch Pathol Lab Med 1986, 110(10):958.

25. World Health Organization (WHO), New Delhi, India: WHO Weekly epidemiological record - Global strategy for further reducing the leprosy burden and sustaining leprosy control activities (plan period 2006-2010). New Delhi, India: $\mathrm{WHO} ; 2010$.

26. Raffe SF, Thapa M, Khadge S, Tamang K, Hagge D, Lockwood DN: Diagnosis and treatment of leprosy reactions in integrated services - the patients' perspective in Nepal. PLoS Negl Trop Dis 2013, 7(3):e2089.

27. Douglas JT, Cellona RV, Fajardo TT, Abalos RM, Balagon MV, Klatser PR: Prospective study of serological conversion as a risk factor for development of leprosy among household contacts. Clin Diagn Lab Immunol 2004, 11:897-900.

28. Bakker MI, Hatta M, Kwenang A, Van Mosseveld P, Faber WR, Klatser PR, Oskam L: Risk factors for developing leprosy--a population-based cohort study in Indonesia. Lepr Rev 2006, 77(1):48-61. Erratum in: Lepr Ver 2006, 77(2):170.

29. World Health Organization (WHO), Geneva, Switzerland: WHO Expert Committee on Leprosy. Geneva, Switzerland: WHO; 1998.

30. Croft RP, Smith WC, Nicholls P, Richardus JH: Sensitivity and specificity of methods of classification of leprosy without use of skin-smear examination. Int J Lepr Other Mycobact Dis 1998, 66(4):445-450.

31. Martelli CM, Stefani MM, Gomes MK, Rebello PF, Peninni S, Narahashi K, Maroclo AL, Costa MB, Silva SA, Sacchetim SC, Nery JA, Salles AM, Gillis TP, Krahenbuhl JL, Andrade AL: Single lesion paucibacillary leprosy: baseline profile of the Brazilian Multicenter Cohort Study. Int J Lepr Other Mycobact Dis 2000, 68(3):247-257.

32. Roche PW, Failbus SS, Britton WJ, Cole R: Rapid method for diagnosis of leprosy by measurements of antibodies to the M. leprae 35-kDa protein: comparison with PGL-I antibodies detected by ELISA and "dipstick" methods. Int J Lepr Other Mycobact Dis 1999, 67(3):279-286.

33. Young DB, Buchanan TM: A serological test for leprosy with a glycolipid specific for Mycobacterium leprae. Science 1983, 221:1057-1059. 
34. Roche PW, Britton WJ, Failbus SS, Neupane KD, Theuvenet WJ: Serological monitoring of the response to chemotherapy in leprosy patients. Int J Lepr Other Mycobact Dis 1993, 61(1):35-43.

35. Baumgart KW, Britton WJ, Mullins RJ, Basten A, Barnetson RS: Subclinical infection with Mycobacterium leprae-a problem for leprosy control strategies. Trans R Soc Trop Med Hyg 1993, 87(4):412-415.

36. Düppre NC, Camacho LA, Sales AM, Illarramendi X, Nery JA, Sampaio EP, Sarno EN, Bührer-Sékula S: Impact of PGL-I seropositivity on the protective effect of BCG vaccination among leprosy contacts: a cohort study. PLoS Negl Trop Dis 2012, 6(6):e1711.

37. Frota CC, Freitas MV, Foss NT, Lima LN, Rodrigues LC, Barreto ML, Kerr LR: Seropositivity to anti-phenolic glycolipid-I in leprosy cases, contacts and no known contacts of leprosy in an endemic and a non-endemic area in northeast Brazil. Trans R Soc Trop Med Hyg 2010, 104(7):490-495.

doi:10.1186/1471-2334-13-497

Cite this article as: Cardoso et al.: Development of a quantitative rapid diagnostic test for multibacillary leprosy using smart phone technology. BMC Infectious Diseases 2013 13:497.

\section{Submit your next manuscript to BioMed Central and take full advantage of:}

- Convenient online submission

- Thorough peer review

- No space constraints or color figure charges

- Immediate publication on acceptance

- Inclusion in PubMed, CAS, Scopus and Google Scholar

- Research which is freely available for redistribution 